\title{
Personalized multi-modal route planning: a preference- measurement and learning-based approach
}

\author{
Jianwei Zhang \\ Eindhoven University of Technology \\ Den Dolech 2 \\ Eindhoven, The Netherlands \\ +31402473315 \\ j.zhang@tue.nt
}

\author{
Theo A. Arentze \\ Eindhoven University of Technology \\ Den Dolech 2 \\ Eindhoven, The Netherlands \\ +31402472283 \\ t.a.arentze@tue.nl
}

\begin{abstract}
Personalized routing recommendation is receiving increasing attention in both academia and engineering. The methodology of how to customize multi-modal routing recommendation to personal preferences of users however is still subject of current research. In the context of the EU FP7 i-Tour project, we developed a set of approaches to solve this problem which are focused on multi-criteria link costs functions, measurement of users' travel preferences and real-time learning of user preferences. The components developed have been successfully integrated and tested as part of the i-Tour prototype system. In this paper we provide an overview of the methods and results.
\end{abstract}

\section{Categories and Subject Descriptors}

I.2.6 [Artificial Intelligence]: Learning; I.6.5 [Simulation and modelling]: Model Development-Modeling methodologies.

\section{General Terms}

Algorithms, Measurement, Performance, Experimentation, Human Factors, Verification.

\section{Keywords}

Traveler information systems, multi-modal route planning, travel preferences, stated choice experiments, multi-criteria costs functions, Bayesian learning.

\section{INTRODUCTION}

In the field of Advanced Traveler Information Systems (ATIS), there is a growing interest in the extension of conventional route planners and navigation systems to consider multimodal routing $[1,2,3,4]$. A multimodal ATIS refers to an approach where networks of different modalities (e.g., car, bike, bus, train) are integrated in a single network. Links interconnecting the networks represent transfers from one mode to another (e.g., parking the car and boarding on a train). The systems are able to generate routes for multimodal trips as well as for uni-modal trips. In this way, multimodal route advice goes beyond the recommendation of routes and also considers the choice of transport mode and possible mode transfers during the trip, such as the use of a Parkand-Ride facility. Furthermore, the integration of activity agenda management and route planning is an objective of a next generation of ATIS. In the area of user-oriented software applications, several calendar or agenda systems have been introduced in recent years, such as for example Google calendar, Microsoft outlook calendar and Yahoo calendar. These systems

Permission to make digital or hard copies of all or part of this work for personal or classroom use is granted without fee provided that copies are not made or distributed for profit or commercial advantage and that copies bear this notice and the full citation on the first page. To copy otherwise, to republish, to post on servers or to redistribute to lists, requires prior specific permission and/or a fee.

I-LOCATE 2014, December 02-04, London, Great Britain

Copyright (C) 2014 ICST 978-1-63190-039-6

DOI 10.4108/icst.mobiquitous.2014.257943 allow users to keep a personal daily activity agenda and obtain alert messages. However, the existing calendar services are not linked to a routing system (for planning and implementing trips) and neither support conflict or constraints checking.

The i-Tour system is a new intelligent traveler information system that offers users routing advice for multimodal trips in urban environments [5]. The system has been developed as an open platform and includes a number of advanced features such as speech recognition, a trust and recommendation system and integrated activity agenda management. Compared to existing traveler information systems, i-Tour uses a comprehensive representation of travel preferences of individual users in a socalled user profile. In the system, user profiles are not static. Based on a built-in learning model i-Tour is able to incrementally learn the personal preferences of a user each time it receives feedback on the travel choice a user makes. Thus, the i-Tour system is adaptive and tailors its advice to the personal preferences of a user incrementally by learning from his or her choice behavior.

In this paper we focus on this latter feature of adaptability to travel preferences of users, which corresponds to a long-standing objective in ATIS development. We give an overview of the components of the i-Tour multi-modal routing system that address this objective. These components relate to various tasks defined in the i-Tour project regarding the routing system. They are described in detail in corresponding research reports and conference and journal papers. Here the main lines of the approach are discussed. To provide a background, the first section focuses on the requirements for multimodal routing and implementation of the basic multimodal routing system. The sections that follow then focus on the innovative features related to travel-advice personalization including multi-criteria link costs functions, user interaction, measurement of user travel preferences and learning of user preferences. Finally, the paper is concluded with a summary of major conclusions.

\section{REQUIREMENTS FOR A MULTIMODAL ROUTING SYSTEM}

\subsection{Some definitions}

Promoting multimodal transport is generally seen as a promising way to alleviate today's transportation problems of deteriorating accessibility of city centers, reduced mobility, and negative impacts of traffic on the environments. Rehrl [1] suggests that to guarantee a high level of mobility in the long term, a shift from 
uni-modal towards multimodal passenger transport is necessary. He also predicts that $20 \%$ of the travelers would be willing to use alternative means of transport if they had access to multimodal travel information.

A mode might be defined by vehicle type or by transport function. The part of the trip where a single mode is used is called a leg. A multimodal trip is a trip where multiple modes are combined so that a trip has multiple legs. A typical example is a trip in which a bicycle is used first to access the railway system, train is used next to travel the main distance and a local bus is used for the final leg from the railway end station to the final destination. A uni-modal trip is the opposite where a single mode is used for the entire trip, such as a private car or a regional train service. In a more narrow definition, which we will use here, a multimodal trip is a trip where a particular private vehicle (car, bike, etc.) is combined with a public transport mode (bus, train, etc.). According to this latter definition, multimodal trips by definition make use of a Park and Ride facility for the vehicle involved in the trip.

The i-Tour routing system should be able to find routes in integrated multimodal networks and, hence, to generate multimodal as well as uni-modal routes for trips. Since the i-Tour routing system considers a multimodal network, transport mode choice is an integral part of route choice in this system.

\subsection{Multi-criteria evaluation and personalized information}

Existing routing systems often consider minimizing travel time as the single objective for determining optimal routes. In reality, however, individuals may wish to take multiple objectives into account: besides travel time, also monetary costs, convenience and possibly environmental impact. Multiple objectives are particularly relevant when multiple modes are considered for a trip. Therefore, a requirement of the i-Tour routing system is that it is able to represent and handle multi-criteria costs functions for transport links. The following criteria are considered relevant:

- Travel time

- Travel costs

- Delays (congestion)

- Risk of delay (travel time reliability)

- Convenience / comfort

- Scenery

- Emission

- Safety

- Generalized costs (combination of the above criteria)

Although convenience and comfort are conceptually different criteria, in practice they are strongly correlated and, hence, they are taken here together as a single dimension. In terms of the road network, convenience may mean that a traveler assigns a higher preference weight to high-level roads (highways) compared to local routes (many turns, mixed traffic, equal level cross-sections, and so on). In terms of public transport, seat availability and avoiding transfers often lead to higher levels of convenience experienced. Generalized costs are defined for transport links as a weighted summation across all criteria using weights that reflect specific preferences of the traveler in the specific situation. Note that this is a broader conceptualization than usually adopted. In the conventional meaning, generalized costs functions include only travel time and travel costs.

It is noted that especially in terms of comfort/-convenience, situational factors tend to play a role. One such condition is the weather: modes such as walking and bicycling tend to be strongly affected by weather conditions. In addition, the activity program and state of the traveler may play a role. For example, it's often nice to walk to office on a not so busy working day, but uncomfortable to walk back home after a busy working day. The i-Tour system currently focuses on travel time, travel costs, convenience/comfort and emission either as single criteria or combined into generalized costs; more criteria can be added, if desired, in future extensions.

Personalization of routing advice (and information) is important to take into account possible differences between persons (e.g., the person has a disability) as well as possible differences between situations (e.g., travelling alone or with a child).

Personalization can be understood on two levels. The first level concerns intrinsic preferences for certain transport modes (e.g., foot, bike or car) or routes (e.g., road A, road B). The second level concerns what was referred to above as multiple criteria, i.e. trade-offs between several objectives (e.g., minimizing travel time, minimizing travel costs, maximizing convenience, minimizing environmental impact). Personalization means that the system takes into account possible differences in preferences between individuals as well as between situations. For example, individuals with tight budgets may give above-average weight to costs, and so on. In order to give personalized advice, the i-Tour routing system should be able to handle different preference schemes (relative weightings of criteria) on above mentioned criteria. The last criterion represents an overall evaluation based on a given preference profile.

\subsection{Environmental friendly travel}

An important objective of the i-Tour personal mobility system is to increase travelers' awareness of the environmental implications of their travel options, in particular, with regard to emission of pollutants that affect global warming (CO2) and air quality (Particular Matter - PM). i-Tour intends to achieve this by providing information about emissions produced by trips made by a user (feedback) and by the travel options he or she may consider before making a trip. i-Tour keeps a track record of each user and gives a reward (e.g., 10\% monthly discount on public transport fares) when a user has achieved some target in terms of emissionsavings by choosing environmental friendly options (e.g., using public transport instead of car). For example, when a user decides to leave the car at home and use public transport for a trip, i-Tour will show the amount of emissions saved and how much still needs to be saved for reaching the target. To provide this functionality, the i-Tour routing system derives emission consequences of routes using the current European standard emission model (COPERT). Because emission is one of the criteria included in evaluation of routes, the routing system does not only give feedback on environmental consequences, but also take these consequences into account in determining an optimal route by incorporating this environmental dimension in generalized link costs functions.

\section{MEASURING USERS' PREFERENCES}

\subsection{Approach}

How the different criteria are weighted in combination with base preferences for particular modes determines a traveler's preference for certain routes and modes. These preferences are not a-priori known. Therefore, to estimate the parameters empirically Kerkman et al. [6] and Arentze and Molin [7] conducted a series of choice experiments using the technique of Conjoint Analysis 
also known as Stated Choice Experiments [8]. In the experiments, subjects are presented hypothetical travel alternatives and are asked to indicate what they would choose if the choice situation were reality. Presented alternatives are constructed in terms of attributes that are relevant for determining a preference (travel time, travel costs, etc.). Based on an experimental design attributes are varied in such a way that the separate effects of the attributes on preference can be determined by statistical analysis of the obtained choice data. Presenting choice tasks may seem more cumbersome than just asking subjects to give preference ratings for individual attributes. However, the advantage of using choice tasks is that individuals are forced to make choices and, hence, to make trade-offs between objectives just as they would do in reality. It has been shown that this leads to more valid measurements of true preferences [9].

Using this methodology, three experiments were designed. Each experiment focuses on a particular domain of travel choice: 1) route choice for a unimodal car trip, 2) choice between car, public-transport and a multimodal option for a trip and 3) choice between different public transport options for a trip. Thus, the experiments cover uni-modal car trips, unimodal public transport trips and multi-modal trips. The experiments were designed in such a way that all preference parameters for the routing system of i-Tour can be estimated. A unique feature of the experiments furthermore is that possible influences of situational factors on preferences were taken into account, to acknowledge the fact that preferences may differ depending on context variables such as purpose of the trip, weather conditions, travel party (traveling alone or with others) and crowdedness. In each experiment a large and representative sample of travelers participated and conducted the choice tasks. The standard multinomial logit model was used to analyze the choice data and estimate the parameters in a random-utility-maximizing framework. We will now discuss the set-up of the three experiments in more detail.

\subsection{The route choice experiment for car trips}

The purpose of this first experiment is to estimate the weights car drivers assign to attributes of routes [6]. The choice tasks presented to participants consistently consisted of two alternative routes and a description of the context for an imaginary trip. Using the stated choice experiment technique, the attributes of choice alternatives as well as relevant context variables are varied based on a factorial design. This design allows an analysis of the relative importance of these attributes for the route choice of car drivers as function of context variables. The attributes used to describe routes are chosen based on existing literature on route choice behavior $[10,11,12]$. Furthermore, the demands of the routing system of $i$-Tour and the possibility of displaying the used attribute information by an in-car navigation system are taken into account when selecting the attributes and the attribute levels. As a result the following attributes were selected:

- Travel time: estimated travel time when there are no delays.

- Estimated delay: predicted extra travel time due to traffic congestion.

- Trip costs: costs defined as sum of fuel costs and a possible road price (or toll).

- Comfort: degree of comfort determined by the number of stops, turns, and intersections on the route and expressed by a quality label.
- Scenery: attractiveness of the landscapes the driver passes through and views along the route, expressed by a quality label.

- Environment and burden: the degree to which the route causes nuisance for local inhabitants as unwanted traffic.

- Safety: degree of safety for the driver determined by presence of intersections, separate lanes, mixed traffic, speed differences and crash barriers.

The choice tasks are presented to the participants of the experiment using an on-line questionnaire. After an explanation of the task, each participant receives a random set of ten choice tasks. In every task, the participant is asked to choose one of the two route options that best reflects his or her preferences. The choice experiment is followed by a subexperiment to reveal the perceptions of the used quality labels as a function of observable route characteristics (not further detailed here). 209 Participants completed the entire questionnaire and the data of these 209 respondents were used in the analysis.

\subsection{The mode choice experiment}

In this second experiment conducted by Arentze and Molin [7], choice tasks consist of three choice alternatives: a car option, a PT option and a car + PT for a trip of approximately $20 \mathrm{~km}$ (PT stands for Public Transport). The attributes correspond to the considerations travelers presumably make when they choose a transport mode: travel time in the different stages (access, main, egress, search and waiting), travel costs (fuel, parking, ticket for public transport), convenience (mode of public transport and transfers) and reliability (possibility of a delay). At the same time, these attributes correspond to the preference parameters that need to be estimated for the routing system of i-Tour. Table 1 shows the specification of attributes and attribute levels used in detail. Context attributes for the trip are varied as well. These include the type of activity for which the trip is made, flexibility in arrival time, travel party, crowdedness, luggage carried and weather conditions. Separate factorial designs were used for creating contexts and choice-sets. Choice tasks are composed by randomly combining a context and a choice-set. 438 individuals participated in the experiment used for estimating the parameters of the i-Tour routing system. Each of them completed 9 choice tasks. Respondents were recruited from an existing large national panel in the Netherlands.

\subsection{The route choice experiment for public- transport trips}

Public transport options may differ on many aspects that are important for determining a choice. The previous experiment already provided insights in evaluations of different travel-time and travel-costs components of public-transport trips. However, these are related to a choice situation where car is also an available option. When a choice for public transport has been made or is forced because a car is not available, a more detailed evaluation of available PT options becomes relevant. In a third experiment conducted by Arentze and Molin [7], all three travel options are public-transport options, but the specific modes differ: the first option is a bus, the second option a local train (many stops) and the third option is an intercity train (few stops and more comfortable seats). The attributes that were varied for each alternative include: in-vehicle travel time, access time, egress time, possibility of delay, transfer time, travel costs, facilities at stations and seat availability. The context was also varied using the same attributes and attribute ranges as in the previous 
experiment. The same factorial design was used to generate contexts. For creating choice-sets, an efficient design was developed based on prior expectations of values of parameters. 672 individuals did the experiment used for the estimation of the i-Tour routing system. They were recruited from the same panel that was also used for the mode choice experiment. Only respondents that used train or some other modes of public transport more than one time a year were considered for this experiment.

Table 1. Attributes of choice alternatives in the mode choice experiment (source [13])

\begin{tabular}{|c|c|c|}
\hline Car alternative & PT alternative & Car + PT alternative \\
\hline main travel time $(20,25,30 \mathrm{~min})$. & mode (bus, local train, intercity train) & PT mode (bus, train, tram) \\
\hline parking search time $(0,5,10 \mathrm{~min})$. & access time $(7,12,17 \mathrm{~min})$. & car detour $(0,-12.5 \%,-25 \%)$ \\
\hline possible delay $(0,10,20 \mathrm{~min})$. & main travel time $(20,25,30 \mathrm{~min})$. & PT travel time $(5,10,15 \mathrm{~min})$. \\
\hline fuel costs - one way $(2,4,6$ Euros $)$ & transfer time (no transfer, 5, $10 \mathrm{~min}$. ) & PT wait time $(5,10,15$ min. $)$ \\
\hline parking costs $(0,5,10$ Euros $)$ & possible delay $(0,10,20 \mathrm{~min})$. & P\&R costs $(0,2,4$ Euros $)$ \\
\hline \multirow{2}{*}{ walk to destination $(0,6,12 \mathrm{~min})}$. & travel costs - one way $(0,2,4$ Euros $)$ & egress time $(2,7,12 \mathrm{~min})$. \\
\hline & egress time $(2,7,12 \mathrm{~min})$. & \\
\hline
\end{tabular}

\subsection{Results of the choice experiments}

The three experiments described above were designed such that all preference parameters required for a multi-modal routing system could be estimated. Willingness-to-pay values for travel time savings that were found are consistent across the experiments and also reasonably in line with findings in the literature although they tend to be somewhat on the high end. The major findings can be summarized as follows (for details see the original sources).

First, travel costs play an important role in the choice behavior. Sensitivity is higher for costs that are clearly visible, such as ticket prices and parking fees, and lower for fuel costs. Travelers for whom car is an option need rather strong compensation before they are willing to use less convenient public transport and Parkand-Ride facilities. The results confirm general experience that only in combination with restrictive parking policies in inner cities, Park-and-Ride use will be considered as an option by car users. Public transport is less attractive when seat availability is uncertain and reliability is limited (possibility of a delay). Walking time in access and egress stages of a trip and transfer time are weighted considerably higher than in-vehicle time. Furthermore, the results indicate that car drivers are to some extent sensitive to environmental consequences of their route choices implying that travel information systems that provide feedback on such consequences can have positive effects for the environment. Finally, it is found that not only socio-demographic differences between persons play a role, but also context variables, such as purpose of the trip, travel party and flexibility in arrival time.

The concrete result of these studies is a set of quantitative preference values that are used to define settings of the parameters of the link costs functions used in the routing system of i-Tour. Using these settings least-costs paths generated by the routing system should match preferences of travelers. Hereby, context variables can be taken into account. For example, when someone is traveling with a young child, an already existing preference for train over bus could become stronger and when a trip is made for leisure purpose willingness to accept somewhat longer travel times could increase. The estimation results provide exact measures for all such trade-offs. Nevertheless individuals may differ in terms of all or some of these preferences. The estimates that were obtained give a good first guess of what the actual preferences of a particular user (of the i-Tour system) will be. The choice experiments can be made available for users of the i-Tour system. Visual versions of the experiments have been developed to make the experiments more appealing and attractive. At registration a new user is invited to conduct the choice tasks. Whenever a user conducts the choice tasks, he or she provides data that the system will use to fine-tune the parameters. Furthermore, based on the learning module described in the next section, the system has the ability to learn user-specific preferences based on feedback on the user's revealed choice behavior.

\section{LEARNING USERS' PERSONAL PREFERENCES}

\subsection{Approach}

The purpose of the learning module is to allow the i-Tour system to incrementally learn the personal preferences of individual users while they use the i-Tour system and reveal their preferences for particular travel options [13]. Based on this learning capability the i-Tour system is able to increasingly tailor its travel advice to the specific preferences of an individual user. Thus the learning component enables the system to realize personalization of advice, which is a major stated objective of a new generation of ATIS.

The learning model developed for the i-Tour system is based on a new approach. The following requirements were formulated for the learning model:

- The system learns personal preferences of users incrementally based on users' revealed choices in choice situations. Each time a choice of a user is revealed the system updates its beliefs about the preferences of that user.

- Preferences relate to the parameters of the evaluation functions (link costs functions) the routing system uses to generate travel advice. The preference parameters are hidden in the sense that they cannot be observed but only inferred from overt behavior.

- The learning method is consistent with principles of Bayesian belief updating. At the same time, the method must be robust and scalable to allow large sets of simultaneous users of the system to be handled simultaneously. 


\section{Hidden \\ preferences $(\theta)$}

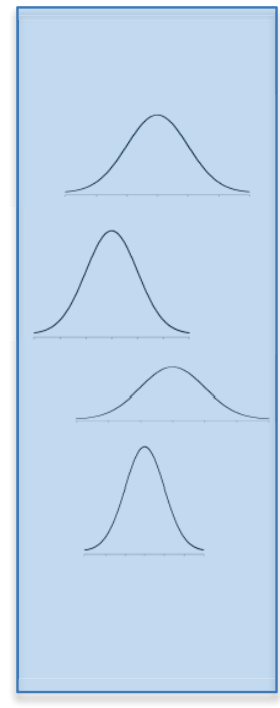

\section{Preference beliefs are adapted}

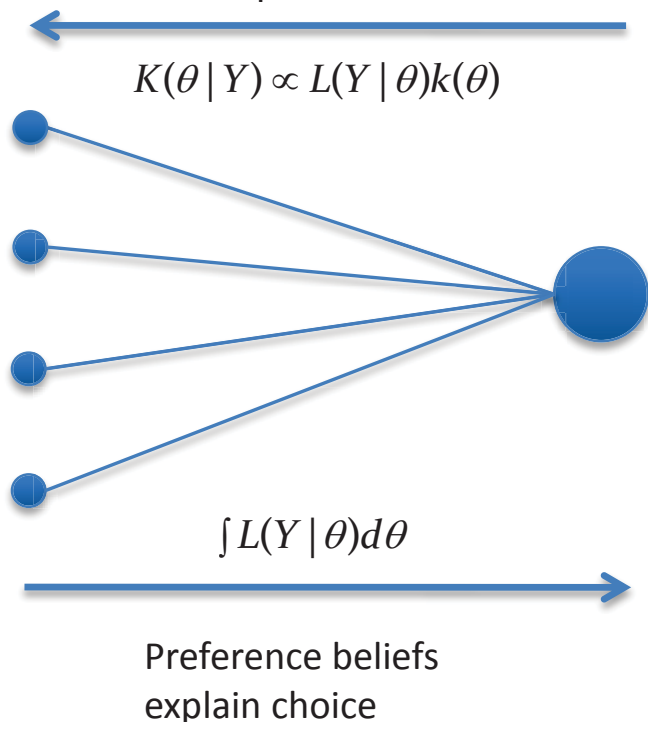

Observed choices $(Y)$

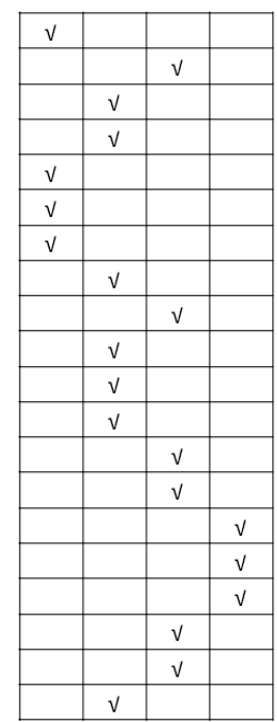

Figure 1. The Bayesian learning model

In the remainder of this section we briefly describe the learning model. The model and results of testing are described in detail in [13].

\subsection{User profile}

A user profile defines values of a pre-defined list of preference parameters. The parameters determine the way a user is supposed to trade-off different objectives such as minimizing travel time, saving travel costs and avoiding inconveniences and effort when he or she can choose between multiple travel options. Technically, the parameters are the constants and coefficients of link costs functions in the multimodal network model the routing system uses to generate routes in a multimodal transport system.

In the system, the parameters are stored as a flat list of items referred to as the Preference table. An example of a Preference table is shown in Table 2. The values shown are based on the combined results of the three experiments. Using these attributes and attribute values allow the link cost functions to be considerably more refined than in existing routing systems. Furthermore, different values apply for different context variables, such as weather and traveling together with a child, and age of the person (senior or not). In the i-Tour database, there is a preference table for each registered user of i-Tour as well as a default specification of the preference table. The default specification corresponds to estimated values based on the experiments; this represents the a-priori beliefs of the system, i.e. the beliefs when no specific information is available for a user. The system learns the preferences based on the choices a user makes at moments when several alternatives are available for a trip (e.g., a public transport and a car-based alternative) and the choice made by the user is revealed to the system. Each time a choice is revealed, the i-Tour system updates its existing beliefs about the users' preferences. The updated beliefs are stored in the Preference table for the user concerned so that on a next occasion the travel options identified by the system will be better adapted to that user's preferences.

\subsection{The learning method}

The learning method is based on the Bayesian method of belief updating. The preference parameters that should be learned by the system are unobserved or hidden variables. What is observed are choices from available alternatives in particular circumstances, whereas what needs to be inferred are preferences in terms of relative weights (values or utilities) the traveler assigns to travel time, travel costs, transport modes and so on. There is a causal relationship between the hidden values and choice outcomes. This causal relationship is defined by the discrete choice model, which is a logit model.

Bayesian belief updating for the case of continuous, multiple variables is intensively studied for the purpose of estimating (preference) parameters of discrete choice models as an alternative to the traditional maximum likelihood methods. In this area, so-called Gibbs sampling is used to obtain draws from a posterior multivariate distribution of parameters $[14,15]$. For the purpose of parameter estimation this method is very powerful. However, for the present purpose of incremental learning it has a serious drawback. The method is computationally very demanding as many thousands of draws are needed to obtain estimates (beliefs) for any set of observations. For incremental learning the sampling procedure would need to be repeated for each single observation making that this method is computationally too demanding. Therefore, an alternative method is developed that requires less computation and yet achieves accurate learning results. 
Table 2. Example of a Preference Table

\begin{tabular}{|c|c|c|c|}
\hline travel time & & & value \\
\hline \multirow[t]{6}{*}{ Car } & base & & -0.06 \\
\hline & road type & Local & -0.015 \\
\hline & & highway & +0.015 \\
\hline & unsafe road & yes $($ no $=0)$ & -0.015 \\
\hline & env. burden & housing (no h. $=0$ ) & -0.015 \\
\hline & purpose & utility (fun = 0) & +0.01 \\
\hline \multirow[t]{2}{*}{ PT } & base & time (min.) & -0.04 \\
\hline & purpose & utility ( fun $=0$ ) & +0.01 \\
\hline \multirow[t]{6}{*}{ bike } & base & time (min.) & -0.1 \\
\hline & heavy bags & yes $($ no $=0)$ & -0.02 \\
\hline & senior & yes $($ no $=0)$ & -0.02 \\
\hline & weather & raining $($ dry $=0)$ & -0.02 \\
\hline & with child & yes $($ no $=0)$ & -0.02 \\
\hline & purpose & utility (fun $=0$ ) & +0.01 \\
\hline \multirow[t]{6}{*}{ walking } & base & time (min.) & -0.15 \\
\hline & heavy bags & yes $($ no $=0)$ & -0.025 \\
\hline & senior & yes $($ no $=0)$ & -0.025 \\
\hline & weather & raining $($ dry $=0)$ & -0.025 \\
\hline & with child & Yes $($ no $=0)$ & -0.025 \\
\hline & purpose & utility (fun $=0$ ) & +0.015 \\
\hline mode & & & value \\
\hline walking & & & 0 \\
\hline bus & & & -3.5 \\
\hline \multirow[t]{3}{*}{ train } & base & & -3.2 \\
\hline & local train & & -0.15 \\
\hline & intercity & & 0.15 \\
\hline ferry & & & -1.5 \\
\hline other PT & & & -3.2 \\
\hline bike & & & -1 \\
\hline & & & -2 \\
\hline Other PV & & & -1.5 \\
\hline Transfer & & & value \\
\hline \multirow[t]{4}{*}{ bike to PT } & base & & 0 \\
\hline & heavy bags & yes $($ no $=0)$ & -0.1 \\
\hline & senior & yes $($ no $=0)$ & -0.1 \\
\hline & purpose & utility ( fun $=0$ ) & 0.1 \\
\hline \multirow[t]{4}{*}{$\begin{array}{l}\text { car to } \mathrm{PT} \\
(\mathrm{P}+\mathrm{R})\end{array}$} & base & & -1.2 \\
\hline & heavy bags & yes $($ no $=0)$ & -0.1 \\
\hline & senior & yes $($ no $=0)$ & -0.1 \\
\hline & purpose & utility ( fun $=0$ ) & 0.1 \\
\hline \multirow[t]{4}{*}{ within PT } & base & & -0.4 \\
\hline & heavy bags & yes $($ no $=0)$ & -0.1 \\
\hline & senior & yes $($ no $=0)$ & -0.1 \\
\hline & purpose & utility ( fun $=0$ ) & 0.1 \\
\hline \multirow[t]{3}{*}{ waiting } & base & time (min.) & -0.1 \\
\hline & with child & yes $($ no $=0)$ & -0.05 \\
\hline & purpose & utility (fun $=0$ ) & 0.05 \\
\hline seat & & & value \\
\hline $\begin{array}{l}\text { seat } \\
\text { availability }\end{array}$ & & $\begin{array}{l}\text { uncertain } \\
(\text { always }=0)\end{array}$ & -0.35 \\
\hline travel costs & & & value \\
\hline PT-ticket & & costs (euro) & -0.2 \\
\hline fuel & & costs (euro) & -0.1 \\
\hline parking fee & & costs (euro) & -0.15 \\
\hline toll & & costs (euro) & -0.4 \\
\hline
\end{tabular}

The proposed new method differs in two ways from existing sampling procedures: 1) belief updating of parameters (in the preference table) is conducted sequentially assuming some order of the parameters when a choice observation is entered to the system and 2) random sampling is replaced by a method of equalinterval sampling from a distribution. This new heuristic method was tested using the data obtained from the choice experiments described above. Since in the experiments repeated choices are observed for each participant separately, the system could be applied to learn the personal preferences incrementally of each individual across the repeated choice observations. The results of this test indicate that the learning model enables the system to improve the prediction of choice behavior considerably for seen cases (around $40 \%$ increase of model fit). Furthermore, an improvement of prediction of unseen (holdout) cases shows that the system has learned something essential about the behavior. Compared to a Gibbs sampling approach the heuristic method learned even faster and nearly as good.

\section{CONCLUSION}

In this paper, we described the multimodal routing system developed for the i-Tour system highlighting the approach used to accomplish adaptability to personal travel preferences of users. We highlighted the specifics of our approach and the innovative properties of the routing system. To summarize, the system contributes to adaptability with the following innovations:

1. A comprehensive representation of travel preferences of users and multiple objectives in multi-criteria link costs functions.

2. Measurement of travel preferences using large-scale choice experiments.

3. An adaptive and personalized system of travel advice using an incremental Bayesian learning model of user's preferences.

The learning model allows the routing system of i-Tour to provide personalized travel information to users. The results of test applications shows that the system rapidly starts to differentiate between users and improves its ability to explain and predict their choice behavior. Using this model, the i-Tour system is able to adapt to personal preferences of users. Initially, it assumes that the user has average preferences. With every observation of a choice of a travel alternative, the system updates the internal user profile (i.e., Preference table). The updated user profile is used in a next occasion when a routing request is posed by that user. In this continuous interactive process, the travel options generated by the system will be increasingly adapted to the preferences of the specific user.

Although separate components have been tested at various test sites, a next step in this R\&D project will involve extensive testing and illustration of the integrated system in full-scale applications.

\section{ACKNOWLEDGMENTS}

i-Tour project is conducted by a consortium including FORMIT Servizi S.p.A., Graphitech Foundation, University College of London, Eindhoven University of Technology, ULA S.r.1., ELASIS S.c.p.A., PTV AG, Cadzow Communications Consulting Ltd, FORMIT Foundation. The research leading to these results has received funding from the European Community's Seventh Framework Program (FP7/2007-2013) under the Grant Agreement 
number 234239. The authors are solely responsible for the information reported in this paper. It does not represent the opinion of the Community. The Community is not responsible for any use that might be made of the information contained in this paper.

\section{REFERENCES}

[1] Rehrl, K., Bruntsch, S. and Mentz, H. (2007). Assisting Multimodal Travellers: Design and Prototypical Implementation of a Personal Travel Companion. IEEE Transactions on Intelligent Transportation Systems. 8(1), 31 42.

[2] Pajor, T. (2009) Multi-modal route planning, Master thesis, University of Karlsruhe.

[3] Liu, L. and Meng, L. (2010). Applying Traditional Speed-up Techniques into Switch- Point-based Multimodal Shortest Paths Algorithms. 6th international conference on Geographic Information Science.

[4] Zhang, L., Li, J., Zhou, K., Gupta, S., Li, M., Zhang, W., Miller, M. and Misener, J. (2011). Design and Implementation of a Traveler Information Tool with Integrated Real-time Transit Information and Multi-modal Trip Planning. In Proceedings of the 90th Annual TRB Meeting.

[5] The i-Tour system website: http://www.itourproject.com/web/

[6] Kerkman, K., T. Arentze, A. Borgers, A. Kemperman (2012) Car Drivers' Compliance with Route Advice and Willingness to Choose Socially Desirable Routes. Transportation research Record, 2012 Vol.1, 102-109.
[7] Arentze, T. and Molin, E. (2013), Travelers' preferences in multimodal networks: design and results of a comprehensive series of choice experiments. In Proceedings of the 92nd Annual TRB Meeting, Washington, D.C.

[8] Louviere, J., D. Hensher, J. Swait (2000). Stated Choice Methods: Analysis and Applications. Cambridge University Press, Cambridge, UK.

[9] Hensher, D. (1994). Stated preference analysis of travel choices: the state of practice. Transportation, 21, 107-133.

[10] Papinski, D. Scott, D. and Doherty, S. (2009). Exploring the route choice decision-making process: A comparison of planned and observed routes obtained using person-based GPS, Transportation Research Part F: Traffic Psychology and Behaviour, 12 (4), 347-358.

[11] Bovy, P. and Stern, E. (1990). Route choice: wayfinding in transport networks, Dordrecht: Kluwer Academic.

[12] Golledge, R. (1999). Wayfinding behavior: cognitive mapping and other spatial processes, Baltimore: John Hopkins University Press.

[13] Arentze, T. (2013), Adaptive, Personalized Travel Information Systems: Bayesian Method to Learn Users' Personal Preferences in Multimodal Transport Networks. IEEE Transactions on Intelligent Transportation Systems, In Press.

[14] Train, K. (2009). Discrete Choice Methods with Simulation, Second Edition. Cambridge University Press, Cambridge.

[15] Rossi, P., Allenby, G. and McCulloch R. (2005). Bayesian Statistics and Marketing. John Wiley \& Sons, West Sussex, UK. 